\title{
Ultrafine Magnetite Nanopowder: Synthesis, Characterization, and Preliminary Use as Filler of Polymethylmethacrylate Nanocomposites
}

\author{
Pietro Russo, ${ }^{1}$ Domenico Acierno, ${ }^{2}$ Mariano Palomba, ${ }^{3}$ Gianfranco Carotenuto, ${ }^{3}$ \\ Roberto Rosa, ${ }^{4}$ Antonino Rizzuti, ${ }^{5}$ and Cristina Leonelli ${ }^{4}$ \\ ${ }^{1}$ Institute of Chemistry and Technology of Polymers, National Research Council of Italy, Via Campi Flegrei, \\ 34-80072 Pozzuoli, Italy \\ ${ }^{2}$ Departments of Materials and Production Engineering, University of Naples Federico II, Piazzale Tecchio, 80-80125 Naples, Italy \\ ${ }^{3}$ Institute for Composite and Biomedical Materials, National Research Council of Italy, Piazzale Tecchio, 80-80125 Naples, Italy \\ ${ }^{4}$ Department of Materials and Environmental Engineering, University of Modena and Reggio Emilia, Strada Vignolese, \\ 905-41125 Modena, Italy \\ ${ }^{5}$ Department of Water Engineering and Chemistry, Polytechnic of Bari, Via Orabona, 4-70125 Bari, Italy
}

Correspondence should be addressed to Pietro Russo, pietro.russo@unina.it

Received 5 April 2012; Revised 28 June 2012; Accepted 10 July 2012

Academic Editor: Menka Jain

Copyright (c) 2012 Pietro Russo et al. This is an open access article distributed under the Creative Commons Attribution License, which permits unrestricted use, distribution, and reproduction in any medium, provided the original work is properly cited.

\begin{abstract}
Magnetite $\left(\mathrm{Fe}_{3} \mathrm{O}_{4}\right)$ nanoparticles prepared by microwave-assisted hydrothermal synthesis have been characterized in terms of morphological and structural features. Electron micrographs collected in both scanning (SEM) and transmission (TEM) modes and evaluations of X-ray powder diffraction (XRD) patterns have indicated the achievement of a monodispersed crystallite structure with particles having an average size around 15-20 nm. Structural investigations by Micro-Raman spectroscopy highlighted the obtainment of magnetite nanocrystals with a partial surface oxidation to maghemite $\left(\gamma\right.$ - $\left.\mathrm{Fe}_{3} \mathrm{O}_{4}\right)$. Preliminary attention has been also paid to the use of these magnetite nanoparticles as filler for a commercial polymethylmethacrylate resin. Hybrid formulations containing up to $3 \mathrm{wt} \%$ of nanoparticles were prepared by melt blending and characterized by calorimetric and thermogravimetric tests. For sake of comparison, same formulations containing commercial $\mathrm{Fe}_{3} \mathrm{O}_{4}$ nanoparticles are also reported. Calorimetric characterization indicates an increase of both glass transition temperature and thermal stability of the nanocomposite systems when loaded with the synthesized magnetite nanoparticles rather then loaded with the same amount of commercial $\mathrm{Fe}_{3} \mathrm{O}_{4}$. This first observation represents just one aspect of the promising potentiality offered by the novel magnetic nanoparticles when mixed with PMMA.
\end{abstract}

\section{Introduction}

In the last decades, a considerable amount of research has been devoted to the synthesis and characterization of iron oxides nanoparticles because of their technological potentialities. In particular, nanosized oxide materials such as magnetite $\left(\mathrm{Fe}_{3} \mathrm{O}_{4}\right)$, maghemite $\left(\gamma-\mathrm{Fe}_{2} \mathrm{O}_{3}\right)$, and hematite $(\alpha$ $\mathrm{Fe}_{2} \mathrm{O}_{3}$ ) have found wide-ranging applications. Hematite nanoparticles, crystallizing in the rhombohedral system and showing a weak ferromagnetic behavior at room temperature [1], have been extensively used as catalyst [2,3], pigment [4], gas sensor [5], optical devices [6], and medicine applications
[7]. Magnetite and maghemite, instead, crystallizing in the cubic crystal system and with a ferromagnetic behavior at room temperature, have been widely exploited in technological applications including information storage [8], sensors [9], refrigeration [10], and coil cores [11], as well as in contrast agent for magnetic resonance imaging $[12,13]$, and potential mediator for magnetic hyperthermia [14].

Among the chemical routes used so far to synthesize iron oxide based nanomaterials, it is worth to mention hydrothermal synthesis $[15,16]$, coprecipitation [17], solgel method [18], and colloidal chemistry method [19]. These methods usually involve synthesizing an iron-based 
precursor gel, followed by decomposing the gel or precursor into the designed crystalline iron oxide phase at an elevated temperature. However, the uniformity of the size of the particle of these nanomaterials was rather poor [20-22].

Considering that chemical composition and morphology controls for these magnetic nanoparticles appear to be crucial for their applications, a specific interest has been dedicated to control the particle size dispersion [23-26]. Small sizes and uniform morphology are typically required for the applications of magnetic nanoparticles because of the dependence of magnetic properties on the morphologies of nanoparticles. When the size of $\mathrm{Fe}_{3} \mathrm{O}_{4}$, nanoparticles is very small, the size of single-magnetic domain is equal to that of each single particle. $\mathrm{Fe}_{3} \mathrm{O}_{4}$ nanoparticles usually exhibit superparamagnetic property [27], therefore, their fabrication is attracting a lot of interest.

Another well-established approach to control, on one side, the morphology of magnetite nanoparticles during their synthesis and, on the other hand, their dispersion in a polymer matrix is the use of suitable polymeric surfactants $[28,29]$. At this regard, given the outstanding interest gained by this class of organic/inorganic materials for applications in many fields, a wide range of resins including polystyrene [30], poly(vinyl alcohol) [31], poly(pyrrole) [32], polyaniline $[33,34]$, and epoxy resins [35] has been already considered.

In this research, ultrafine magnetite nanoparticles were synthesized by an innovative hydrothermal microwaveassisted route and systematically characterized in terms of morphological and structural aspects. Literature references $[36,37]$ employ iron(III) chloride, $\mathrm{FeCl}_{3} \cdot 6 \mathrm{H}_{2} \mathrm{O}$ as the metal precursor in the hydrolytic reactions, while in the present work the $\mathrm{Fe}_{3} \mathrm{O}_{4}$ precursor was $\mathrm{FeCl}_{2} \cdot 4 \mathrm{H}_{2} \mathrm{O}$. This is a main difference that requires to be highlighted since most of the "soft-chemistry" synthetic strategies (both hydrolytic and nonhydrolytic) for the obtainment of magnetite nanoparticles start from iron-(III-)based precursor (see e.g., $[38,39]$ ). Moreover, in the work by Parsons et al. [37] the product obtained at the reaction temperature of $100^{\circ} \mathrm{C}$ and $125^{\circ} \mathrm{C}$ was an iron (III) oxyhydroxide chloride, commonly known as akaganeite. An increase in the reaction temperature up to $250^{\circ} \mathrm{C}$ led to single hematite phase. Thus, the work by Pearsons and coworkers substantially differs from ours in both the precursors used and the synthesised products.

As concerns the paper by Wang et al. [36], a further difference (apart from the use of an iron(III) precursor) with respect to our paper is represented by the use of polyethylene glycol (PEG) as surfactant. Indeed, those authors employed such a surfactant in order to obtain nanoparticles of phase pure $\mathrm{Fe}_{3} \mathrm{O}_{4}$; however, as they noticed (through FTIR study), the surfactant was adsorbed on the surface of nanoparticles product thus affecting the saturation magnetization.

In order to emphasize potential applications of the novel magnetic powder preliminary attention has been devoted to polymer nanocomposites based on an amorphous thermoplastic matrix. In particular, given the relevant interest gained so far by polymer systems containing magnetic particles in many different industrial areas (e.g., radio-absorbing materials, polymer welding, etc. $[40,41]$, synthesized magnetite was loaded in a polymethylmethacrylate (PMMA) by using a conventional melt-blending process.

Compounds with contents equal to 1 and $3 \%$ by weight of new magnetite were compared with analogous systems filled with commercial magnetite nanopowder in terms of thermal parameters such as glass transition and thermal stability of the matrix. At this regards, results were discussed taking the neat matrix, processed under the same conditions, as the reference material.

\section{Experimental}

2.1. Materials. The polymeric matrix used in this study was the polymethylmethacrylate (PMMA) Optix CA-51 (MFI $15 \mathrm{~g} / 10 \mathrm{~min}$ ) supplied by Plaskolite-Continental Acrylics.

Commercial magnetite nanopowder (named $\mathrm{Fe}_{3} \mathrm{O}_{4}(\mathrm{C}$ ) in the text and figures) was provided by Sigma Aldrich with an average particle size less than $50 \mu \mathrm{m}$ and a BET surface area higher than $60 \mathrm{~m}^{2} / \mathrm{g}$.

Hydrazine hydrate and $\mathrm{NaOH}$ (pellets) were purchased from Sigma Aldrich, while $\mathrm{FeCl}_{2} \cdot 4 \mathrm{H}_{2} \mathrm{O}$ was provided by Merck. All of these precursors for the microwave-assisted hydrothermal synthesis of magnetite nanoparticles were of analytical grade and used without any purification.

2.2. Synthesis of Magnetite Nanoparticles $\left(\mathrm{Fe}_{3} \mathrm{O}_{4}(\mathrm{~S})\right)$. The general procedure for the microwave assisted hydrothermal synthesis of $\mathrm{Fe}_{3} \mathrm{O}_{4}$ nanoparticles is as follows: $0.5150 \mathrm{~g}$ $(2.6 \mathrm{mmol})$ of $\mathrm{FeCl}_{2} \cdot 4 \mathrm{H}_{2} \mathrm{O}$ were dissolved in $13 \mathrm{~mL}$ of deionized water under nitrogen atmosphere, in a Teflonlined digestion vessel. A previously prepared $\mathrm{NaOH} /$ hydrate hydrazine solution $(0.2609 \mathrm{~g}(6.5 \mathrm{mmol})$ of $\mathrm{NaOH}$ in $2.174 \mathrm{~mL}(70 \mathrm{mmol})$ of $\left.\mathrm{N}_{2} \mathrm{H}_{4} \cdot \mathrm{H}_{2} \mathrm{O}\right)$ was dropwise added to the iron (II) solution under nitrogen and vigorous magnetic stirring.

The sealed reaction vessel was hydrothermally treated using a microwave digestion system ETHOS TC (Milestone) operating at $2.45 \mathrm{GHz}$ frequency, at $100^{\circ} \mathrm{C}$ for $120 \mathrm{~min}$. with a continuous and accurate computer controlling of reaction time, temperature, pressure, and microwave power. After the reaction the product was naturally cooled to room temperature, and the obtained dark-brown powder was centrifuged (4000 rpm), washed several times with deionized water, and finally dried overnight at $80^{\circ} \mathrm{C}$, under vacuum. Surface area was determined to be $81 \mathrm{~m}^{2} / \mathrm{g}$.

2.3. Preparation of $\mathrm{PMMA} / \mathrm{Fe}_{3} \mathrm{O}_{4}$ Nanocomposites. The inclusion of nanosized particles of magnetite in the molten PMMA was carried out in a HAAKE Minilab Rheomex microcompounder mod. CTW5 conical double-screw extruder. In this apparatus, an integrated bypass valve enables the recirculation of the melt via a back flow channel. In our case, pellets of PMMA and proper amounts of magnetite were fed to the microcompounder in a maximum time of 2 minutes, allowing a recirculation of the molten compound for 5 minutes before collecting the extruded composite systems.

A flat temperature profile of $220^{\circ} \mathrm{C}$ and a screw speed of $80 \mathrm{rpm}$ were employed operating in corotating mode. 


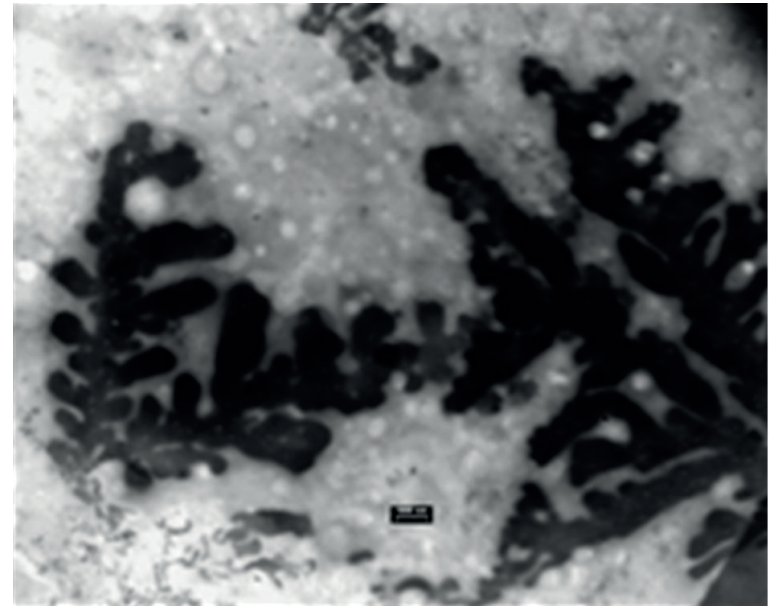

(a)

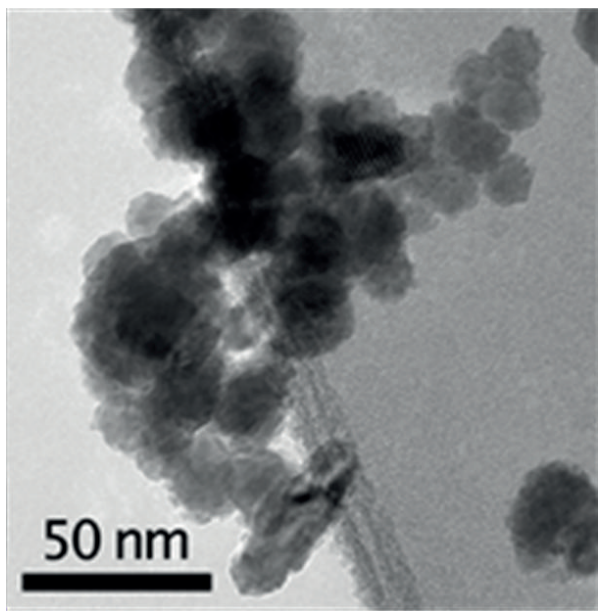

(b)

FIGURE 1: TEM micrograph showing the characteristic dendritic structure generated by the magnetite nanoparticles (left-marker: 1000 nm), and detail of the microscopic structure (right).

2.4. Characterization Techniques. Nanostructured magnetite morphology was imaged by transmission electron microscopy (TEM, Philips EM2085, $100 \mathrm{kV}$ ) and scanning electron microscopy (SEM, Cambridge-S360). In particular, in the preparation of specimens for the TEM investigation, the magnetite powder was dispersed in ethanol by applying ultrasounds (ultrasonic bath, Bandeline RK515CH), and a polymeric surfactant (poly (vinyl pyrrolidone), $M_{w}=10,000 \mathrm{gmol}^{-1}$ ) was added in order to achieve a stable colloid. Then, a drop of this colloidal suspension was placed on a TEM copper grid coated by Formvar resin thin film and allowed to dry in air at room temperature. After drying, the copper grid was graphitized by sputtering. The crystallographic structure of magnetite was investigated by large angle X-ray powder diffraction (XRD, X'Pert $\mathrm{PRO}, \mathrm{PANAlytical}, \mathrm{CuK}_{\alpha}$ radiation). The structure of the magnetite powder was also investigated by Micro-Raman spectroscopy (LabRam, Horiba Jobin-Yvon, Longjumeau, France) employing a $632.81 \mathrm{~nm}$ wavelength laser which was used on both magnetite powders. In addition, we employed a D1 type filter in order to use $2 \mathrm{~mW}$ output laser power, focused onto the sample using a $10 \mathrm{x}$ objective lens, in order to avoid any phase transformation of the analyzed powders, as a consequence of laser-induced thermal effects. It is well known that under heat treatment magnetite easily transforms into the oxidized crystal of maghemite at around $200^{\circ} \mathrm{C}$ to finally complete oxidation to hematite $\left(\alpha-\mathrm{Fe}_{2} \mathrm{O}_{3}\right)$ at around $400^{\circ} \mathrm{C}[42]$.

About PMMA-based nanocomposites, melt-blended products were analysed by differential scanning calorimetry using a DSC (DSC1, Mettler Toledo Instrument). Samples were run by a heating rate of $20^{\circ} \mathrm{C} / \mathrm{min}$ on the temperature range from 25 to $200^{\circ} \mathrm{C}$ with the aim to verify any influence of magnetite nanoparticles on the glass transition temperature of nanocomposites.

Further thermogravimetric tests were carried out by using a Q5000-TA Instrument. Approximately 15 mg of each material were submitted to a temperature ramp from 25 to $600^{\circ} \mathrm{C}$ at a heating rate of $10^{\circ} \mathrm{C} / \mathrm{min}$ under nitrogen. The influence on magnetite nanoparticles on thermal stability and decomposition behavior of polymer matrix were investigated taking the unfilled PMMA as the reference material.

\section{Result and Discussion}

The as-synthesized magnetite $\left(\mathrm{Fe}_{3} \mathrm{O}_{4}\right)$ was made of a very fine dark-brown powder, which exhibited a good magnetic behavior (Figure 1). As reported in a previous work [43], magnetism of the probe was measured by means of MPMSR5S-SQUID magnetometer of Quantum-Design. In the ZFC/FC curves the Verwey transition at $120 \mathrm{~K}$ was observed. This transition justified the nanoparticle core constituted by stoichiometric magnetite [44]. Magnetization-temperature curves show a saturation magnetization of about $64 \mathrm{emu} / \mathrm{g}$ which therefore much lower than the bulk value of magnetite $92 \mathrm{emu} / \mathrm{g}$, observing additional remanence and coercivity. The decrease of the saturation magnetization is, probably, due to the maghemite shell surrounding the nanoparticles.

According to the morphological investigation using transmission electron microscope (TEM), the as-prepared powder consisted of monodispersed nanoparticles with a size of ca. $15-20 \mathrm{~nm}$. As visible in the TEM micrograph given in Figure 1 (left side, magnification 25000x), these particles produced very large branched or dendritic structures. Details observed at high magnification $(180000 x)$ confirmed that the sample is constituted by pseudospherical nanoparticles with a monomodal particle size distribution.

$\mathrm{Fe}_{3} \mathrm{O}_{4}$ (s) sample was characterized also by scanning electron microscopy (SEM), and according to Figure 2 the nanoparticles appearas aggregates in densely packed secondary particles. Moreover, at higher enlargements (Figure 2(a)right) these secondary particles appear as powder grains made of closely packed spherical nanoparticles partially sintered together. 

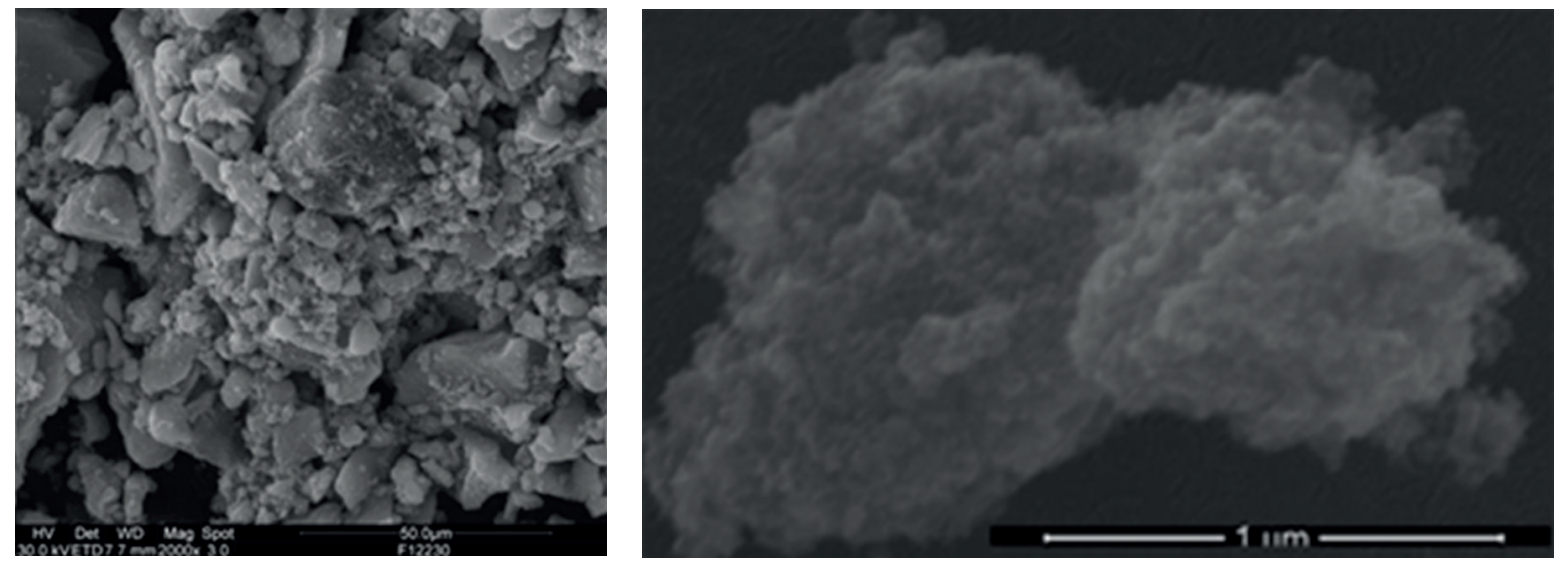

(a)

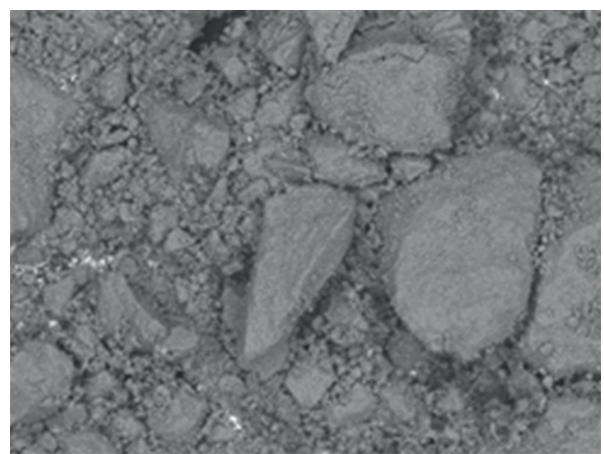

$50 \mu \mathrm{m}$

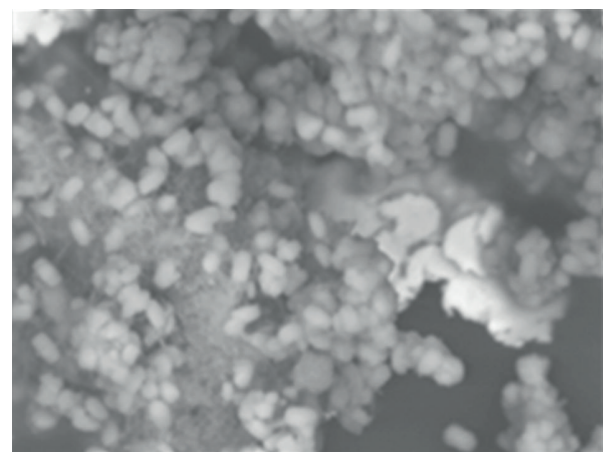

$1 \mu \mathrm{m}$

(b)

FIGURE 2: SEM micrograph of the as-synthesized powder ((a)-left), and detail of the single grain ((a)-right) and of the commercial powder at low ((b)-left) and high ((a)-right) enlargements.

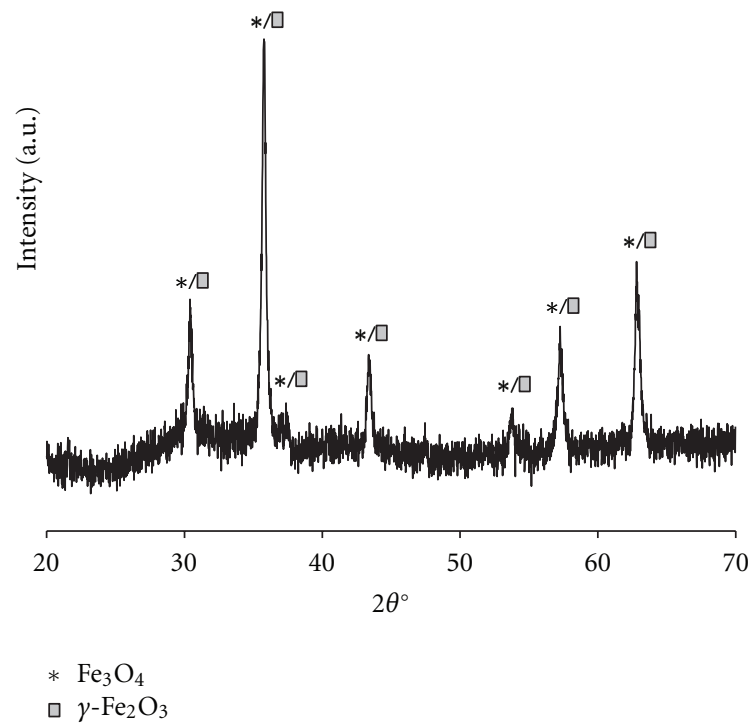

FIGURE 3: XRD pattern of the as-prepared magnetite nanoparticles.
Microstructure of commercial iron oxide powders has been also characterized by means of scanning electron microscopy. Backscattered electron (BSE) image is reported in Figure 2(b). The average grain size is in the $0.8-2.5 \mu \mathrm{m}$ size. Differently from $\mathrm{Fe}_{3} \mathrm{O}_{4}$ hydrothermal powder the commercial one does not present an enhanced nanostructure.

As visible in Figure 3, X-ray diffractogram (XRD) of the as synthesized samples showed a diffraction pattern characteristic of magnetite $\left(\mathrm{Fe}_{3} \mathrm{O}_{4}\right)$. In particular, the peaks at $2 \theta$ equal to $30.1^{\circ}, 35.4^{\circ}, 43.1^{\circ}, 53.4^{\circ}, 57.1^{\circ}$, and $62.6^{\circ}$ can be indexed as (220), (311), (400), (511), and (440) lattice planes of cubic magnetite, respectively (JCPDS 19-629). The average $\mathrm{Fe}_{3} \mathrm{O}_{4}$ crystallite size was calculated by applying the Debye-Scherrer equation $\left(D_{\mathrm{hkl}}=0.9 \lambda / \beta \cos \theta\right.$, in which $\beta$ stands for the half-width of the XRD diffraction line, $\theta$ is the peak position in angles, and $\lambda$ is the $\mathrm{CuK}_{\alpha}$ radiation wavelength, that is $1.54056 \AA$ ) to the reflection peak (311) and it resulted approximately of $10 \mathrm{~nm}$. Such crystallite size approximately corresponds to the TEM (Figure 1, right) average size of particles, which indicates a monocrystalline nature of the particles. 


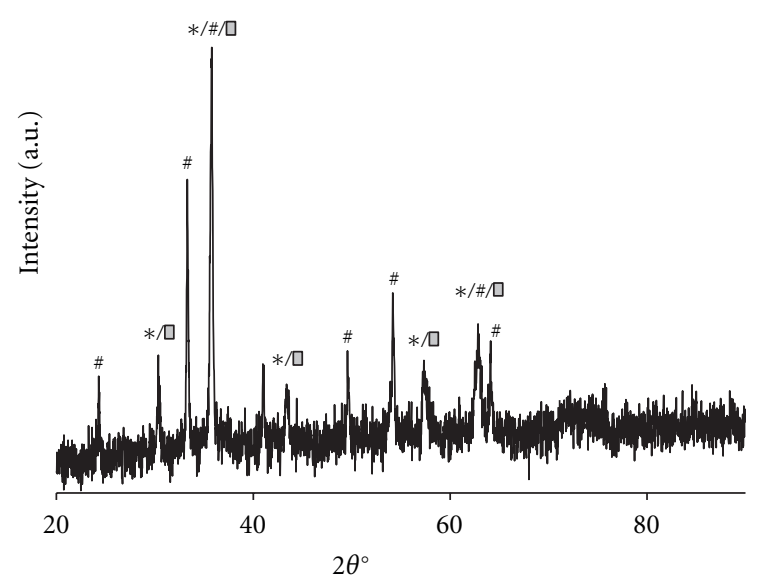

* $\mathrm{Fe}_{3} \mathrm{O}_{4}$

\# $\alpha-\mathrm{Fe}_{2} \mathrm{O}_{3}$

ㄱ $\gamma-\mathrm{Fe}_{2} \mathrm{O}_{3}$

FIgURE 4: XRD pattern of the commercial magnetite nanostructured particles.

The presence of hematite, beside to the major magnetite phase, has been confirmed by X-ray diffraction analysis on the commercial iron oxide nanopowders (Figure 4).

To evaluate the purity of crystalline phases in the two magnetite powders, a specific characterization procedure was set up.

The XRD patterns of magnetite $\left(\mathrm{Fe}_{3} \mathrm{O}_{4}\right)$ and maghemite $\left(\gamma-\mathrm{Fe}_{2} \mathrm{O}_{3}\right)$ differ only for the presence of a few diffraction peaks with intensity lower than $5 \%$. Thus, the absence of these low-intensity peaks, from the X-ray diffractogram of Figure 4, can not be considered a proof of the obtainment of pure magnetite phase during the microwave assisted hydrothermal synthesis [40]. An alternative technique has been envisaged in Raman spectroscopy that is considered to be a powerful tool to distinguish the different structural phases of iron oxides [43].

Raman spectra of both commercial and synthetic iron oxide nanopowders are reported in Figure 5 (please notice that the $y$-axis is not the same for both spectra).

The presence of nanostructured crystalline powders in the sample of hydrothermally prepared magnetite is clearly visible from the broadness of the peaks in Figure 5(a). Magnetite phase is easily discernible by its main band centred at around $667 \mathrm{~cm}^{-1}$. Thus, magnetite can be assumed to be present in both commercial and synthetic iron oxide nanopowders. In the Raman spectrum of as-prepared magnetite nanoparticles, typical maghemite band $\left(720 \mathrm{~cm}^{-1}[44\right.$, $45]$ ) is clearly evident, thus confirming the obtainment of magnetite nanocrystals with the surface partially oxidised to maghemite. Accordingly to [46] maghemite phase should show an additional band at $511 \mathrm{~cm}^{-1}$, which could be either the one centred at $460 \mathrm{~cm}^{-1}$ or could be identified as the minor peak at its right.

On the other hand, commercial iron oxide nanoparticles consist of a complex mixture of magnetite, maghemite, and hematite phases. The latter can be easily individualised by the

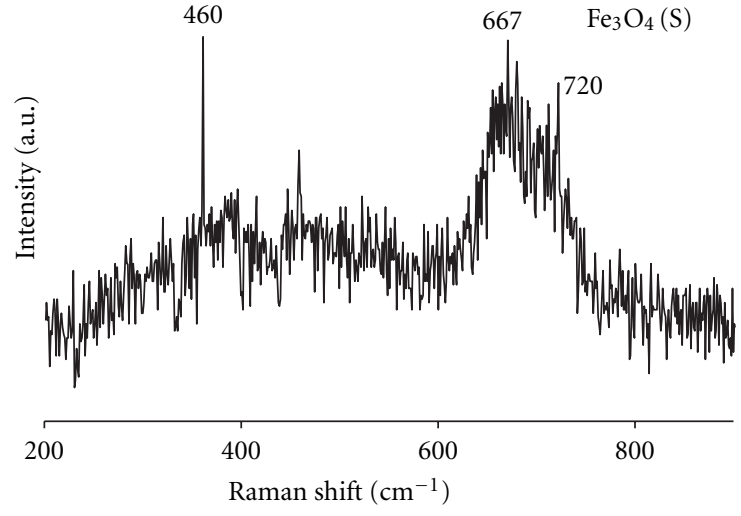

(a)

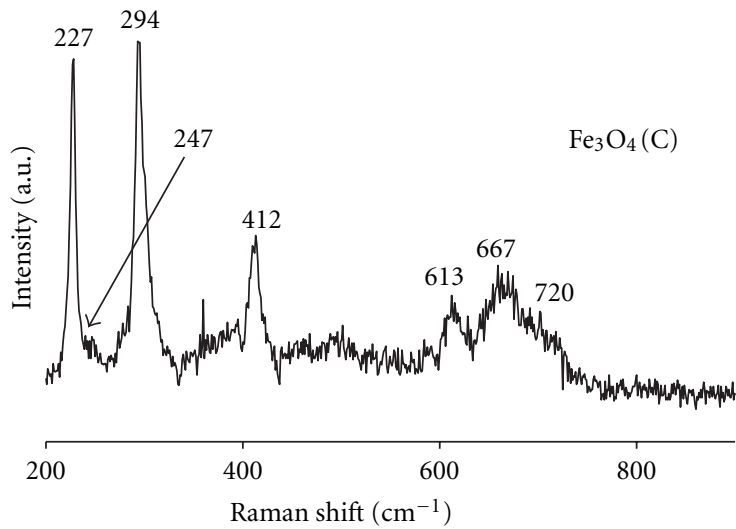

(b)

FIGURE 5: Raman spectra of as-prepared magnetite nanoparticles (a) and the commercial magnetite nanostructured particles (b).

bands situated at 227, 247, 294, 412, and $613 \mathrm{~cm}^{-1}$, according to literature $[44,45]$. The presence of hematite phase has been also confirmed by X-ray diffraction analysis on the commercial iron oxide nanopowders (Figure 4).

Regarding thermal properties of melt-blended nanocomposite systems based on polymethylmethacrylate, PMMA, in Figure 6, the comparison of thermograms collected by calorimetric tests is depicted. In particular, a clear shift of the inflection point usually associated with the glass-rubber transition is observed for all hybrid formulations.

The analysis showed an increase of the glass transition temperature $T_{\mathrm{g}}$, evaluated as the temperature at the inflection point of the thermograms. This parameter appeared to increase with the filler content especially in presence of synthesized magnetite nanoparticles (denoted as S sample), with respect to commercial powders (denoted as $\mathrm{C}$ samples).

This behavior is usually ascribed to restrictions of the polymer chains mobility by the inclusion of rigid nanoparticles. Moreover, islands of nanoparticles may adsorb portion of adjacent chains, especially if longer than the average size of macromolecules constituting the matrix and, thus, having the highest relaxation times, enhancing this effect.

Appreciating the evaluations shown in Figure 5 in the light of the range of experimental error evaluated on at 
TABLe 1: Thermal parameters of PMMA-based materials ( $H_{\max }$ : DTG peak height; FWMH: full width at medium height of DTG peak).

\begin{tabular}{lcccc}
\hline Material & $T_{-5 \%}\left({ }^{\circ} \mathrm{C}\right)$ & $T_{\text {peak }}$ DTG $\left({ }^{\circ} \mathrm{C}\right)$ & $H_{\max }$ & FWMH $\left({ }^{\circ} \mathrm{C}\right)$ \\
\hline Neat extruded PMMA & $355.2 \pm 0.5$ & $379.1 \pm 0.7$ & 45 & 42 \\
PMMA/1 wt $\% \mathrm{Fe}_{3} \mathrm{O}_{4}(\mathrm{C})$ & $357.8 \pm 1.2$ & $397.4 \pm 0.9$ & 48 & 46 \\
PMMA/3 wt $\% \mathrm{Fe}_{3} \mathrm{O}_{4}(\mathrm{C})$ & $358.8 \pm 0.7$ & $393.8 \pm 0.6$ & 38 & 45 \\
PMMA/1 wt $\% \mathrm{Fe}_{3} \mathrm{O}_{4}(\mathrm{~S})$ & $358.1 \pm 0.8$ & $395.1 \pm 0.3$ & 38 & 47 \\
PMMA/3 wt $\% \mathrm{Fe}_{3} \mathrm{O}_{4}(\mathrm{~S})$ & $359.4 \pm 0.7$ & $395.5 \pm 0.4$ & & 38 \\
\hline
\end{tabular}

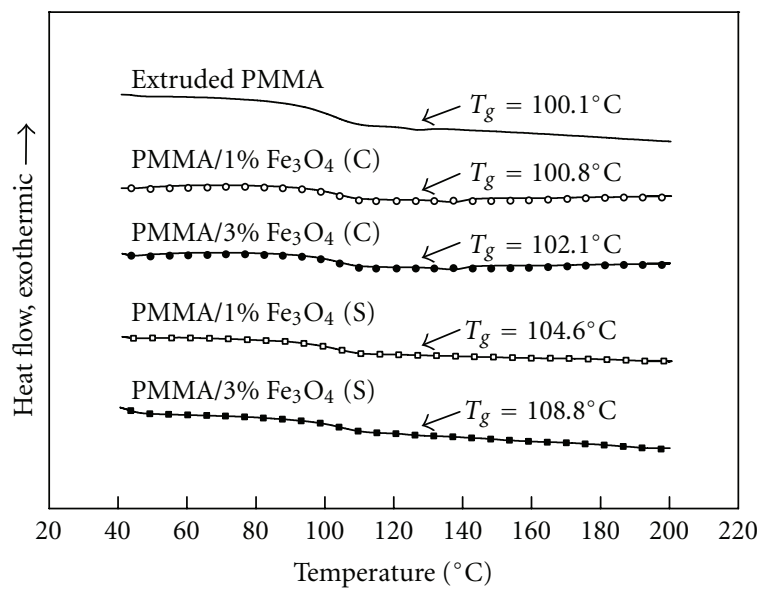

FIGURE 6: Comparison of DSC curves of the nanocomposites based on PMMA taking the neat matrix as reference material.

least 5 tests for each material and approximately equal to $2 \%$, it can be stated that the commercial magnetite does not significantly alter the glass transition temperature of the matrix, at least on the interval compositions examined so far. On the contrary, the $\mathrm{Tg}$ is changed from $100.1^{\circ} \mathrm{C}$ for the neat matrix to 104.6 and $108.8^{\circ} \mathrm{C}$ for nanocomposites containing 1 and $3 \mathrm{wt} \%$, respectively, of microwave hydrothermally synthesized magnetite. On the basis of the above considerations, these benefits can be explained by assuming the establishment of specific chemical-physical interactions at the filler-matrix interface such as to improve the thermal performance of the amorphous matrix.

Finally, Figures 7 and 8 report thermogravimetric (TG) mass loss curves and their first derivative, respectively, for all PMMA nanocomposites.

In more details, looking to the data, averaged on at least 3 tests for each material and summarized in Table 1, the following considerations may be drawn.

Assuming the temperature at which a $5 \%$ of weight loss is achieved $\left(T_{-5 \%}\right)$ as the thermal stability of the material, a slight increase of this parameter can be observed for all nanocomposite systems with respect to the neat matrix. This effect can be ascribed to the restrictions of the polymer chains mobility already invoked to explain the increase of the glass transition temperature as well as to the absorption of polymer on the oxide surface by methoxycarbonyl groups $\left(-\mathrm{C}(\mathrm{O}) \mathrm{OCH}_{3}\right)[47]$.

Moreover, in all cases, the residue at $600^{\circ} \mathrm{C}$ is comparable with the amount of magnetite nanoparticles, initially included in the PMMA.
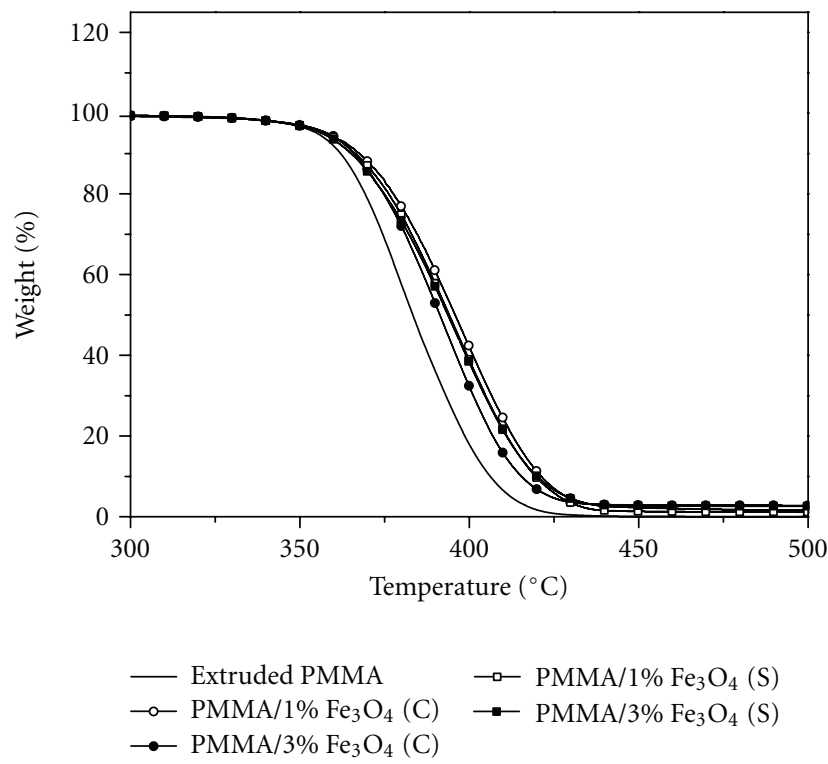

FIGURE 7: TG mass loss curves of all investigated materials under $\mathrm{N}_{2}$. (Heating rate $10 \% \mathrm{~min}$ ).

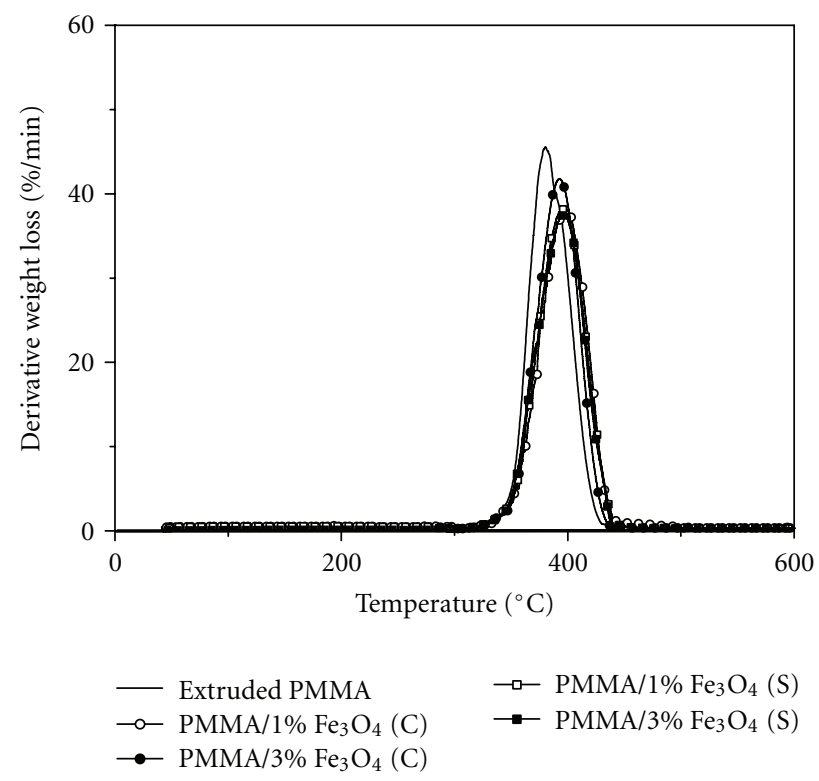

FIGURE 8: Derivative TG curves of all investigated materials under $\mathrm{N}_{2}$. (Heating rate $10^{\circ} / \mathrm{min}$ ). 
The comparison of derivative signals in Figure 8, instead, has allowed to assess any effect related to the presence of magnetite nanoparticles on the thermal behaviour of investigated materials. Starting from the evidence showing the existence of a single decomposition signal for both unfilled matrix and nanocomposite systems, additions of magnetite always induced a shift of the maximum decomposition signal ( $T_{\text {peak }}$ DTG) to higher temperature with respect to the reference neat matrix (see Table 1). In addition, the hybrid formulations showed a reduction in the height of the decomposition signal.

This behavior appeared to be influenced by the type of magnetite used. In particular, for samples containing $1 \%$ by weight of nanoparticles a reduction in the height of the signal equal to about $15 \%$ compared to the reference material was shown regardless of the type of included magnetite: as shown in Table 1, the height of the decomposition signal goes from 45 to $38^{\circ} \mathrm{C}$. But further increase of the content of filler implied an inversion of the trend for systems containing commercial magnetite $\left(\mathrm{Fe}_{3} \mathrm{O}_{4}(\mathrm{C})\right)$, with a reduction in the lowering of the signal compared to the reference value equal to approximately $9 \%$ (from the reference value 45 to $41^{\circ} \mathrm{C}$ ). Instead, no further effects were observed in presence of synthesized filler $\left(\mathrm{Fe}_{3} \mathrm{O}_{4}(\mathrm{~S})\right)$.

Finally, a slight widening of the decomposition signal was observed for all hybrid formulations with respect to unfilled PMMA and evaluated in terms of full width at medium height (FWMH) (see Table 1).

These phenomena, probably related to the influence of filler on involved combustion processes, are still under investigation.

\section{Conclusions}

The microwave hydrothermally prepared nanoparticles of magnetite are compared to the commercial ones in terms of effects on the thermal properties of PMMA nanocomposites. The smaller particles size of the synthesized nanoparticles with respect to the commercial ones and the absence of agglomeration allows a better dispersion in the polymeric matrix via extrusion process.

The effects of nanoparticles dispersion in the PMMA have been measured in terms of glass transition temperature and thermal stability of melt-blended systems compared with the unfilled matrix.

The experimental results showed an increase of both of these parameters especially in the case of new nanoparticles. This effect, attributed to specific filler-matrix interactions, probably more efficient for the greater uniformity of size distribution of synthesized magnetites with respect to the commercial ones, highlights their interesting potentialities of use.

Finally, being aware that a magnetic characterization of the nanocomposite systems is as important as the morphological and thermal ones, authors are also expecting better magnetic properties in the $\mathrm{Fe}_{3} \mathrm{O}_{4}$-synthetic_PMMA samples.

\section{Acknowledgments}

Authors are particularly grateful to Prof. Pietro Baraldi, Department of Chemistry, University of Modena and Reggio
Emilia, for useful discussion on Raman spectra and to Kilian Pollok, Bayerisches Geoinstitut, Universität Bayreuth, Germany, for providing one of the TEM images.

\section{References}

[1] Z. Jing and S. Wu, "Synthesis and characterization of monodisperse hematite nanoparticles modified by surfactants via hydrothermal approach," Materials Letters, vol. 58, no. 27-28, pp. 3637-3640, 2004.

[2] Z. Zhong, J. Lin, S. P. Teh, J. Teo, and F. M. Dautzenberg, "A rapid and efficient method to deposit gold particles on catalyst supports and its application for CO oxidation at low temperatures," Advanced Functional Materials, vol. 17, no. 8, pp. 14021408, 2007.

[3] A. P. Kozlova, S. Sugiyama, A. I. Kozlov, K. Asakura, and Y. Iwasawa, "Iron-oxide supported gold catalysts derived from gold-phosphine complex $\mathrm{Au}(\mathrm{PPh} 3)(\mathrm{NO} 3)$ : state and structure of the support," Journal of Catalysis, vol. 176, no. 2, pp. 426438, 1998.

[4] D. Hradil, T. Grygar, J. Hradilová, and P. Bezdička, "Clay and iron oxide pigments in the history of painting," Applied Clay Science, vol. 22, no. 5, pp. 223-236, 2003.

[5] X. Q. Liu, S. W. Tao, and Y. S. Shen, "Preparation and characterization of nanocrystalline $\alpha-\mathrm{Fe}_{2} \mathrm{O}_{3}$ by a sol-gel process," Sensors and Actuators B, vol. 40, no. 2-3, pp. 161-165, 1997.

[6] M. J. Espin, A. V. Delgado, and J. D. G. Durán, "Optical properties of dilute hematite/silicone oil suspensions under low electric fields," Journal of Colloid and Interface Science, vol. 287, no. 1, pp. 351-359, 2005.

[7] S. Laurent, D. Forge, M. Port et al., "Magnetic iron oxide nanoparticles: synthesis, stabilization, vectorization, physicochemical characterizations and biological applications," Chemical Reviews, vol. 108, no. 6, pp. 2064-2110, 2008.

[8] A. H. Lu, E. L. Salabas, and F. Schüth, "Magnetic nanoparticles: synthesis, protection, functionalization, and application," Angewandte Chemie, vol. 46, no. 8, pp. 1222-1244, 2007.

[9] J. Zhang, S. Rana, R. S. Srivastava, and R. D. K. Misra, "On the chemical synthesis and drug delivery response of folate receptor-activated, polyethylene glycol-functionalized magnetite nanoparticles," Acta Biomaterialia, vol. 4, no. 1, pp. 4048, 2008.

[10] C. Flesch, C. Delaite, P. Dumas, E. Bourgeat-Lami, and E. Duguet, "Grafting of poly( $\varepsilon$-caprolactone) onto maghemite nanoparticles," Journal of Polymer Science A, vol. 42, no. 23, pp. 6011-6020, 2004.

[11] J. Sun, S. Zhou, P. Hou et al., "Synthesis and characterization of biocompatible $\mathrm{Fe}_{3} \mathrm{O}_{4}$ nanoparticles," Journal of Biomedical Materials Research A, vol. 80, no. 2, pp. 333-341, 2007.

[12] C.-M. Lee, H.-J. Jeong, E.-M. Kim et al., "Synthesis and characterization of iron oxide nanoparticles decorated with carboxymethyl curdlan," Macromolecular Research, vol. 17, no. 2, pp. 133-136, 2009.

[13] S. Wada, K. Tazawa, I. Furuta, and H. Nagae, "Antitumor effect of new local hyperthermia using dextran magnetite complex in hamster tongue carcinoma," Oral Diseases, vol. 9, no. 4, pp. 218-223, 2003.

[14] V. Sreeja and P. A. Joy, "Microwave-hydrothermal synthesis of $\gamma$ - $\mathrm{Fe}_{2} \mathrm{O}_{3}$ nanoparticles and their magnetic properties," Materials Research Bulletin, vol. 42, no. 8, pp. 1570-1576, 2007.

[15] T. J. Daou, G. Pourroy, S. Bégin-Colin et al., "Hydrothermal synthesis of monodisperse magnetite nanoparticles," Chemistry of Materials, vol. 18, no. 18, pp. 4399-4404, 2006. 
[16] K. Simeonidis, S. Mourdikoudis, M. Moulla et al., "Controlled synthesis and phase characterization of Fe-based nanoparticles obtained by thermal decomposition," Journal of Magnetism and Magnetic Materials, vol. 316, no. 2, pp. e1-e4, 2007.

[17] I. Nedkov, T. Merodiiska, L. Slavov, R. E. Vandenberghe, Y. Kusano, and J. Takada, "Surface oxidation, size and shape of nano-sized magnetite obtained by co-precipitation," Journal of Magnetism and Magnetic Materials, vol. 300, no. 2, pp. 358367, 2006.

[18] J. Hu, G. Chen, and I. M. C. Lo, "Removal and recovery of $\mathrm{Cr}(\mathrm{VI})$ from wastewater by maghemite nanoparticles," Water Research, vol. 39, no. 18, pp. 4528-4536, 2005.

[19] P. D. Cozzoli, E. Snoeck, M. A. Garcia et al., "Colloidal synthesis and characterization of tetrapod-shaped magnetic nanocrystals," Nano Letters, vol. 6, no. 9, pp. 1966-1972, 2006.

[20] P. Berger, N. B. Adelman, K. J. Beckman, D. J. Campbell, A. B. Ellis, and G. C. Lisensky, "Preparation and properties of an aqueous ferrofluid," Journal of Chemical Education, vol. 76, no. 7, pp. 943-948, 1999.

[21] S. C. Gu, T. Shiratori, and M. Konno, "Synthesis of monodisperse, magnetic latex particles with polystyrene core," Colloid and Polymer Science, vol. 281, no. 11, pp. 1076-1081, 2003.

[22] T. Belin, N. Guigue-Millot, T. Caillot, D. Aymes, and J. C. Niepce, "Influence of grain size, oxygen stoichiometry, and synthesis conditions on the $\gamma-\mathrm{Fe}_{2} \mathrm{O}_{3}$ vacancies ordering and lattice parameters," Journal of Solid State Chemistry, vol. 163, no. 2, pp. 459-465, 2002.

[23] S. Sun and H. Zeng, "Size-controlled synthesis of magnetite nanoparticles," Journal of the American Chemical Society, vol. 124, no. 28, pp. 8204-8205, 2002.

[24] I. Nyiro-Kosa, D. C. Nagy, and M. Pósfai, "Size and shape control of precipitated magnetite nanoparticles," European Journal of Mineralogy, vol. 21, no. 2, pp. 293-302, 2009.

[25] N. Mizutani, T. Iwasaki, S. Watano, T. Yanagida, and T. Kawai, "Size control of magnetite nanoparticles in hydrothermal synthesis by coexistence of lactate and sulfate ions," Current Applied Physics, vol. 10, no. 3, pp. 801-806, 2010.

[26] D. Shi, J. P. Cheng, F. Liu, and X. B. Zhang, "Controlling the size and size distribution of magnetite nanoparticles on carbon nanotubes," Journal of Alloys and Compounds, vol. 502, no. 2, pp. 365-370, 2010.

[27] X. T. Wen, J. X. Yang, B. He, and Z. Gu, "Preparation of monodisperse magnetite nanoparticles under mild conditions," Current Applied Physics, vol. 8, no. 5, pp. 535-541, 2008.

[28] X. Wang, C. Zhang, X. Wang, and H. Gu, "The study on magnetite particles coated with bilayer surfactants," Applied Surface Science, vol. 253, no. 18, pp. 7516-7521, 2007.

[29] P. Guardia, B. Batlle-Brugal, A. G. Roca et al., "Surfactant effects in magnetite nanoparticles of controlled size," Journal of Magnetism and Magnetic Materials, vol. 316, no. 2, pp. e756e759, 2007.

[30] L. Jiang, W. Sun, and J. Kim, "Preparation and characterization of $\omega$-functionalized polystyrene-magnetite nanocomposites," Materials Chemistry and Physics, vol. 101, no. 2-3, pp. 291-296, 2007.

[31] A. K. Bajpai and R. Gupta, "Synthesis and characterization of magnetite (Fe3O 4)polyvinyl alcohol-based Nanocomposites and study of superparamagnetism," Polymer Composites, vol. 31, no. 2, pp. 245-255, 2010.

[32] S.-J. Yen, E.-C. Chen, R.-K. Chiang, and T.-M. Wu, "Preparation and characterization of polypyrrole/magnetite nanocomposites synthesized by in situ chemical oxidative polymerization," Journal of Polymer Science B, vol. 46, no. 13, pp. 12911300, 2008.
[33] J. H. Kim, F. F. Fang, H. J. Choi, and Y. Seo, "Magnetic composites of conducting polyaniline/nano-sized magnetite and their magnetorheology," Materials Letters, vol. 62, no. 1718, pp. 2897-2899, 2008.

[34] H. Gu, Y. Huang, X. Zhang et al., "Magnetoresistive polyaniline-magnetite nanocomposites with negative dielectric properties," Polymer, vol. 53, no. 3, pp. 801-809, 2012.

[35] L. A. Ramajo, A. A. Cristóbal, P. M. Botta, J. M. Porto López, M. M. Reboredo, and M. S. Castro, "Dielectric and magnetic response of $\mathrm{Fe}_{3} \mathrm{O}_{4}$ /epoxy composites," Composites A, vol. 40, no. 4, pp. 388-393, 2009.

[36] W. W. Wang, Y. J. Zhu, and M. L. Ruan, "Microwave-assisted synthesis and magnetic property of magnetite and hematite nanoparticles," Journal of Nanoparticle Research, vol. 9, no. 3, pp. 419-426, 2007.

[37] J. G. Parsons, C. Luna, C. E. Botez, J. Elizalde, and J. L. Gardea-Torresdey, "Microwave-assisted synthesis of iron(III) oxyhydroxides/oxides characterized using transmission electron microscopy, X-ray diffraction, and X-ray absorption spectroscopy," Journal of Physics and Chemistry of Solids, vol. 70, no. 3-4, pp. 555-560, 2009.

[38] X. Yang, W. Jiang, L. Liu et al., “One-step hydrothermal synthesis of highly water-soluble secondary structural $\mathrm{Fe}_{3} \mathrm{O}_{4}$ nanoparticles," Journal of Magnetism and Magnetic Materials, vol. 324, no. 14, pp. 2249-2257, 2012.

[39] M. Niederberger and G. Garnweitner, "Organic reaction pathways in the nonaqueous synthesis of metal oxide nanoparticles," Chemistry, vol. 12, no. 28, pp. 7282-7302, 2006.

[40] A. V. Mitin, V. A. Tarasov, V. N. Doronin, and R. A. Nazipov, "Magnetic nanostructured polymer composites," Physics of the Solid State, vol. 53, no. 12, pp. 2336-2339, 2011.

[41] L. E. Nita, A. P. Chiriac, I. Neamtu, and C. Vasile, "Magnetic composites obtainment based on styrene polymers," Journal of Applied Polymer Science, vol. 100, no. 5, pp. 4133-4141, 2006.

[42] N. Pinna, S. Grancharov, P. Beato, P. Bonville, M. Antonietti, and M. Niederberger, "Magnetite nanocrystals: nonaqueous synthesis, characterization, and solubility," Chemistry of Materials, vol. 17, no. 11, pp. 3044-3049, 2005.

[43] B. Nigrovski, A. Rizzuti, C. Leonelli, B. Ondruschka, and P. Scholz, "Preparation of magnetite nanoparticles via microwave-assisted hydrothermal process," in Proceedings of the WG 003/04 COST D32 Action, Chemistry under High-Energy Microenvironments, Final Meeting, Krakow, Poland, September 2009.

[44] R. Aragón, J. P. Shepherd, J. W. Koenitzer, D. J. Buttrey, R. J. Rasmussen, and J. M. Honig, "Influence of nonstoichiometry on the Verwey transition in Fe 3(1- $\delta) \mathrm{O} 4$," Journal of Applied Physics, vol. 57, no. 8, pp. 3221-3222, 1985.

[45] O. N. Shebanova and P. Lazor, "Raman study of magnetite $\left(\mathrm{Fe}_{3} \mathrm{O}_{4}\right)$ : laser-induced thermal effects and oxidation," Journal of Raman Spectroscopy, vol. 34, no. 11, pp. 845-852, 2003.

[46] A. M. Jubb and H. C. Allen, "Vibrational spectroscopic characterization of hematite, maghemite, and magnetite thin films produced by vapor deposition," ACS Applied Materials and Interfaces, vol. 2, no. 10, pp. 2804-2812, 2010.

[47] A. Laachachi, M. Cochez, M. Ferriol, J. M. Lopez-Cuesta, and E. Leroy, "Influence of $\mathrm{TiO}_{2}$ and $\mathrm{Fe}_{2} \mathrm{O}_{3}$ fillers on the thermal properties of poly(methyl methacrylate) (PMMA)," Materials Letters, vol. 59, no. 1, pp. 36-39, 2005. 

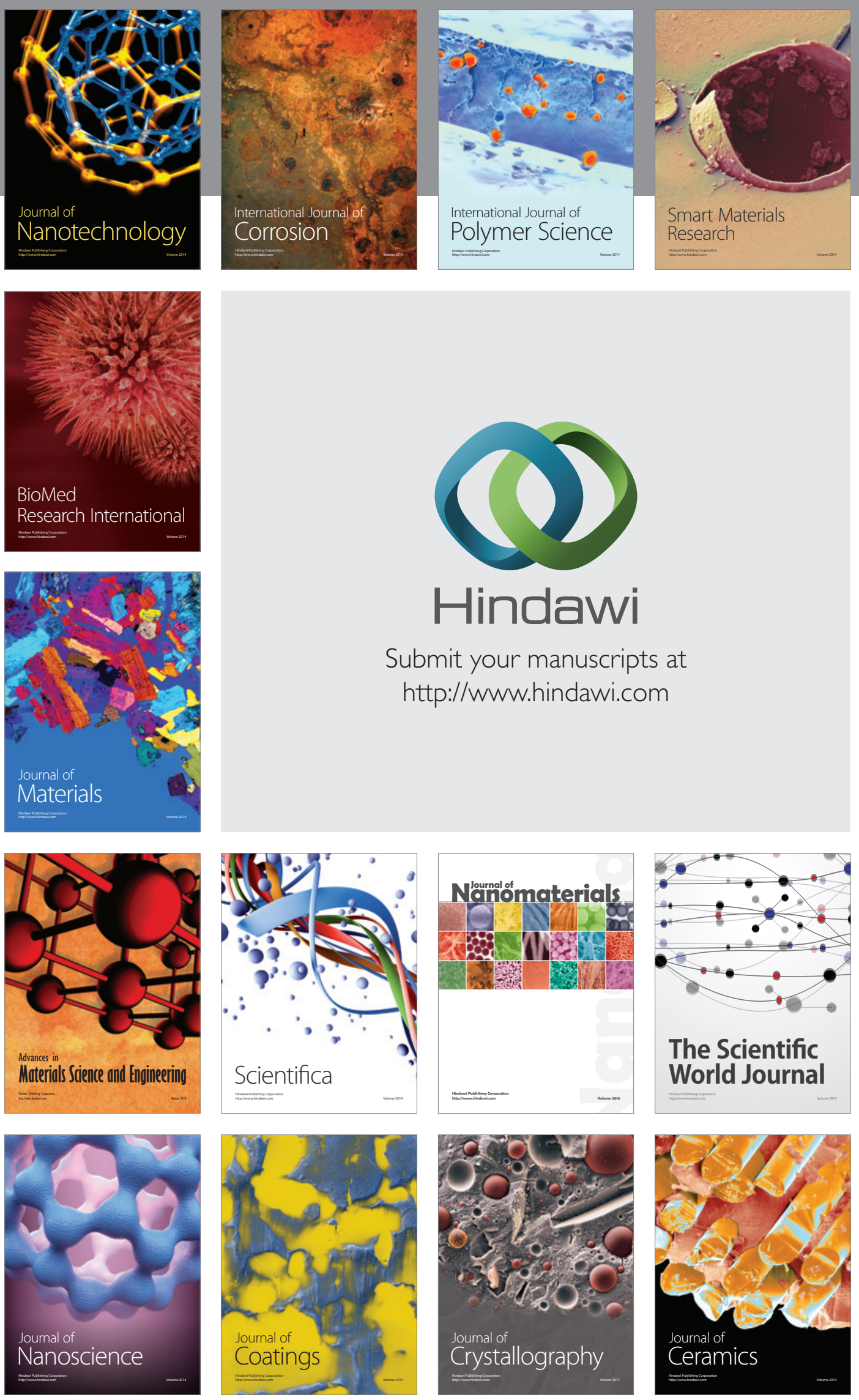

The Scientific World Journal

Submit your manuscripts at

http://www.hindawi.com

\section{World Journal}

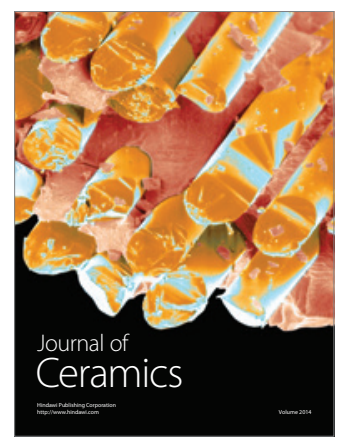

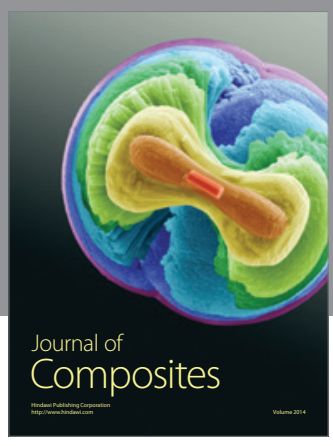
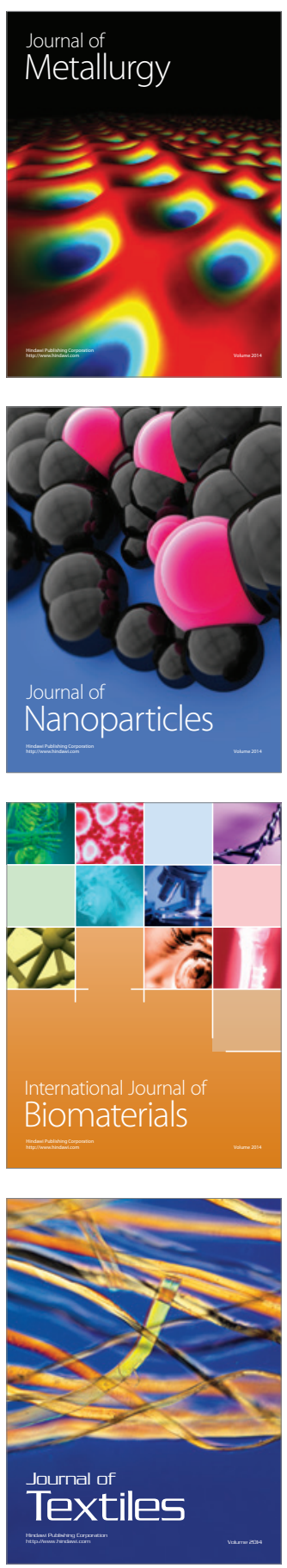